\title{
Conservation laws and symmetries in stochastic thermodynamics
}

\author{
Matteo Polettini, Gregory Bulnes-Cuetara, and Massimiliano Esposito \\ Complex Systems and Statistical Mechanics, Physics and Materials Science Research Unit, University of Luxembourg, \\ Campus Limpertsberg, 162a avenue de la Faïencerie, L-1511 Luxembourg (G. D. Luxembourg)
}

(Received 21 February 2016; revised manuscript received 4 May 2016; published 10 November 2016)

\begin{abstract}
Phenomenological nonequilibrium thermodynamics describes how fluxes of conserved quantities, such as matter, energy, and charge, flow from outer reservoirs across a system and how they irreversibly degrade from one form to another. Stochastic thermodynamics is formulated in terms of probability fluxes circulating in the system's configuration space. The consistency of the two frameworks is granted by the condition of local detailed balance, which specifies the amount of physical quantities exchanged with the reservoirs during single transitions between configurations. We demonstrate that the topology of the configuration space crucially determines the number of independent thermodynamic affinities (forces) that the reservoirs generate across the system and provides a general algorithm that produces the fundamental affinities and their conjugate currents contributing to the total dissipation, based on the interplay between macroscopic conservations laws for the currents and microscopic symmetries of the affinities.
\end{abstract}

DOI: 10.1103/PhysRevE.94.052117

\section{INTRODUCTION}

Thermodynamics is the science of nonequilibrium processes occurring in open systems that interact with an environment. Today, a dramatic evolution is reshaping it, from a patchwork of general principles and applied laws - and a riddle for students, from a pedagogical perspective-to a systematic and comprehensive theory called stochastic thermodynamics (ST), where all propositions are well founded on the mathematics of Markov processes [1-4], with a span of applications ranging from molecular motors [5] to photovoltaic cells [6] and beyond. Still some conceptual leaps need to be filled before this program can be deemed complete. According to classic formulations $[7,8]$, phenomenological thermodynamics is a discourse about physical currents of energy, matter, charge, etc.; their conservation; and their degree of degradation, quantified by the entropy production rate (EPR). The conceptual pathway to nonequilibrium processes starts from an ideally isolated universe, where Noether's theorem states that conservation laws follow from symmetries of the dynamics. Nonequilibrium behavior ensues when one can separate the universe into a system and its environment, which is eventually structured into several competing ideal reservoirs that always remain at equilibrium. The system's effective dynamics becomes dissipative, but, as we will argue, its features still bear the signature of the conservation laws across the system-environment interface.

More specifically, let us consider a "black box" scenario where we only know that a system is in a nonequilibrium steady state generated by $R$ reservoirs denoted $r$ and described by $n_{Y}$ different affinities $f_{y}$ (intensive thermodynamic variables such as inverse temperatures $\beta_{r}$ or chemical potentials $-\beta_{r} \mu_{r}$ ), which we list in a vector $\left|f_{Y}\right\rangle$. From phenomenological thermodynamics, the EPR of this setup quantifies the entropy changes in the reservoirs caused by the $n_{Y}$ physical currents $j_{y}$ of conserved quantity $y$ conjugated to the affinities (currents of extensive quantities such as energy $-\dot{\epsilon}_{r}$ or matter $-\dot{n}_{r}$ ). By convention these currents enter the reservoirs and are listed as a vector $\left|j_{Y}\right\rangle$. According to the fundamental relation, each conjugate pair $f_{y} j_{y}$ is a contribution to the entropy change of a reservoir, meaning that their sum over all $n_{Y}$ pairs is the physical EPR, denoted $\sigma_{Y}=\left\langle f_{Y} \mid j_{Y}\right\rangle \geqslant 0$. At this level of description, besides global conservation laws for like currents (e.g., $\sum_{r} \dot{\epsilon}_{r}=0$ or $\sum_{r} \dot{n}_{r}=0$ ), no other argument can be used to further simplify the EPR. It will thus display a number of currents and affinities equal to at most $n_{Y}$ minus the number of conserved quantities.

ST instead allows us to "enter the box." It describes the system degrees of freedom as nodes of a network and its dynamics as a Markov process driven by transition probability rates, associated to network edges. Pairs of nodes may be connected by multiple edges when different mechanisms (i.e., sets of reservoirs) trigger the transition. At that level, the only conservation law is that of probability and the dynamics is characterized by the statistical EPR which additively measures the breakage of detailed balance in each edge of the network, and will be denoted $\sigma_{X}=\left\langle f_{X} \mid j_{X}\right\rangle \geqslant 0$, where $\left\langle f_{X}\right|$ and $\left|j_{X}\right\rangle$ are, respectively, vectors of edge affinities and currents. The crucial ingredient connecting the statistical and physical levels of description in ST is the local detailed balance (LDB) condition $[1,9,10]$. It relates rates to the physical quantities exchanged with the reservoirs in such a way that $\sigma_{X}$ will eventually be solely expressed in terms of physical currents. However, identifying the fundamental currents and affinities that contribute to the EPR can only be done "by hand" in very simple systems and no systematic procedure exists to address this crucial question in more complex ones. An important step forward was made by Schnakenberg [11], who showed that the statistical EPR can be expressed as a sum over the number of fundamental cycles $n_{X}$ of the network, of products of the cycle affinities $F_{X}$ and currents $J_{X}$, i.e., $\sigma_{X}=\left\langle F_{X} \mid J_{X}\right\rangle$. Alas, the number of configuration cycles typically grows exponentially large with the network size, and, most importantly, many of these cycle affinities are not independent of each other. It is then crucial to overcome this major limitation in Schnakenberg's analysis.

In this paper, by adapting the formalism of closed and open chemical networks proposed in Ref. [12], we provide a general and systematic procedure for doing so. Beside its conceptual aspects, bringing light on the trade-off between 
symmetries and conservation laws - a mechanism that is somewhat reminiscent of the Noether theorem-our procedure paves the way to applications of ST to systems with arbitrarily large and complex networks. In the next section we present our main results with the aid of a simple example; in the following we introduce another example to illustrate the potential of the theory to be scaled up to networks of arbitrary complexity. Finally, before drawing conclusions, we demonstrate how the fluctuation theorem for fundamental physical currents arises.

\section{MAIN RESULT}

We start by illustrating our argument using a simple setup. The system consists of two single-level quantum dots, coupled among themselves by an effective capacitance $C$, and exchanging energy and particles with three electronic reservoirs $r=1,2,3$, each at different inverse temperatures $\beta_{r}$ and chemical potentials $\mu_{r}$, according to Fig. 1(a), for a total of $n_{Y}=6$ affinities. The system's dynamics can be described as a continuous-time Markov jump process with rates describing transitions between states $x$ in the system space of configurations $X=\{00,01,10,11\}$ which correspond to the absorption or emission of energy and particles from the reservoirs, as depicted in Fig. 1(b) [13-15]. Each possible transition mechanism belongs to the space of the oriented edges of a network (or graph) that has $X$ as its vertices [see Fig. 1(c)]. The rates satisfy local detailed balance and a simple stochastic thermodynamics ensues. When regarding the system as a "black box" that only serves to process energy fluxes $\dot{\epsilon}_{r}$ and particle fluxes $\dot{n}_{r}$ between the reservoirs, physical EPR reads $\sigma_{Y}=-\sum_{r=1}^{3} \beta_{r}\left(\dot{\epsilon}_{r}-\mu_{r} \dot{n}_{r}\right)=\left\langle f_{Y} \mid j_{Y}\right\rangle$, where the current and affinity vectors are, respectively, $\left|j_{Y}\right\rangle=\left(-\dot{\epsilon}_{r},-\dot{n}_{r}\right)$ and $\left\langle f_{Y}\right|=\left(\beta_{r},-\mu_{r} \beta_{r}\right)$. One can now make use of conservation of energy $\dot{\epsilon}_{1}+\dot{\epsilon}_{2}+\dot{\epsilon}_{3}=0$ and of particles $\dot{n}_{1}+\dot{n}_{2}+\dot{n}_{3}=0$ to simplify the EPR as $\sigma_{Y}=\sum_{r=1}^{2}\left[\left(\beta_{3}-\beta_{r}\right) \dot{\epsilon}_{r}+\left(\beta_{r} \mu_{r}-\right.\right.$ $\left.\left.\beta_{3} \mu_{3}\right) \dot{n}_{r}\right]$ and thus reduce the number of affinities from $n_{Y}=6$ to 4 . The "black box" perspective does not allow us to go further. However, when considering the internal structure of the system, one notices that electrons cannot cross the condensator. Hence there is one additional conservation law $\dot{n}_{1}=0$ which allows us to further simplify the EPR by reducing one more affinity,

$$
\begin{aligned}
\sigma_{Y} & =\sum_{r=1}^{2}\left(\beta_{3}-\beta_{r}\right) \dot{\epsilon}_{r}+\left(\beta_{2} \mu_{2}-\beta_{3} \mu_{3}\right) \dot{n}_{2} \\
& =\left\langle F_{Y} \mid J_{Y}\right\rangle .
\end{aligned}
$$

We thus learned that of the $n_{Y}=6$ affinities describing the reservoirs, only three fundamental affinities $\left\langle F_{Y}\right|$ and their conjugated currents $\left\langle J_{Y}\right|$ ultimately control the physical EPR, due to two global conservation laws and one system-specific conservation law. While identifying the latter was easy in this simple setup, doing so in more complex systems is not and requires a systematic procedure.

We now proceed with the general theory. We consider a system dynamics described by the master equation $\partial_{t} p_{x}=$ $\sum_{v, x^{\prime}}\left(w_{x x^{\prime}}^{v} p_{x^{\prime}}-w_{x^{\prime} x}^{v} p_{x}\right)$, where $v$ distinguishes between different transitions that connect two states. The network EPR $\sigma_{X}$ is defined as [11]

$$
\sigma_{X}=\frac{1}{2} \sum_{x, x^{\prime}, v} \overbrace{\left(w_{x x^{\prime}}^{v} p_{x^{\prime}}-w_{x^{\prime} x}^{v} p_{x}\right)}^{j_{x x^{\prime}, v}^{X}} \overbrace{\ln \frac{w_{x x^{\prime}}^{v} p_{x^{\prime}}}{w_{x^{\prime} x}^{v} p_{x}}}^{f_{x x^{\prime}, v}^{X}} \geqslant 0,
$$

where the overbraces, respectively, define the probabilistic currents and their conjugate affinities. Letting $e=\left(x x^{\prime}, v\right)_{x<x^{\prime}}$ label the edges of the graph, the incidence matrix $A^{X}$ of the network has entries

$$
A_{x, e}^{X}= \begin{cases}+1 & \text { if } \stackrel{e}{\longrightarrow} x \\ -1 & \text { if } \stackrel{e}{\leftrightarrows} x \\ 0 & \text { otherwise }\end{cases}
$$

The master equation can then be cast in the form of a continuity equation $\partial_{t}|p\rangle=A^{X}\left|j_{X}\right\rangle$. We will focus on steady states, where Kirchhoff's current law holds $A^{X}\left|j_{X}\right\rangle=0$, implying that $\left|j_{X}\right\rangle$ lives in the null space of the incidence matrix, which is known to be spanned by $n_{X}$ independent cycles of the graph. Schnakenberg [11] described a procedure (that we call routine 1, see Refs. [11,16,17] for details) to find a basis of cycle vectors. The steady network currents can be expressed as $\left|j_{X}\right\rangle=A^{C}\left|J_{X}\right\rangle$, where $A^{C}$ is a full-rank matrix of independent null vectors of $A^{X}$, viz. $A^{X} A^{C}=0$, and $\left|J_{X}\right\rangle$ is a vector of coefficients with the meaning of independent cycle currents. Notice that there is a certain degree of freedom in the choice (a)

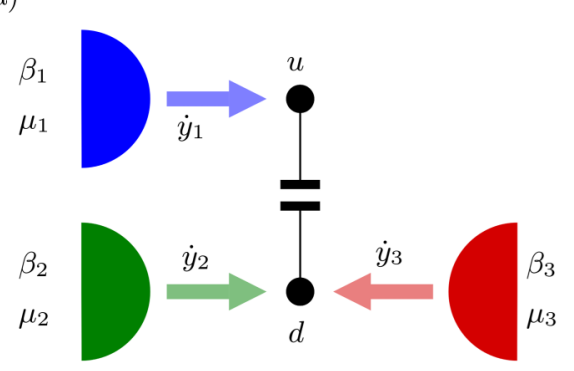

(b)

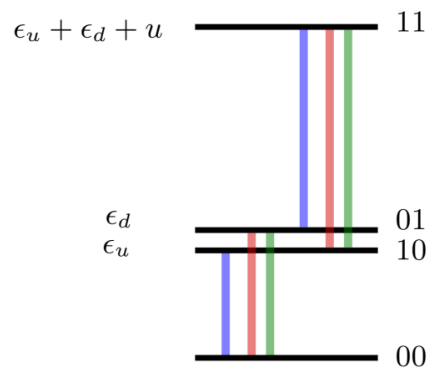

(c)

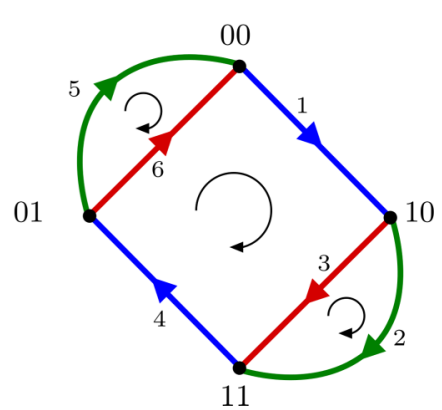

FIG. 1. (a) Physical representation of two quantum dots capacitively coupled and in contact with three reservoirs, $\dot{y}_{r}=\left(\dot{\epsilon}_{r}, \dot{n}_{r}\right)$; $($ b) many-body energy levels and their single-level occupation numbers; (c) network representation of the configuration space where the Markov process occurs, with an arbitrary orientation assigned to each edge. 
TABLE I. Recap of pairs of conjugate observables.

\begin{tabular}{|c|c|}
\hline $\begin{array}{c}\left\langle f_{Y}|,| j_{X}\right\rangle \\
\left\langle F_{X}|,| J_{X}\right\rangle \\
\left\langle f_{Y}|,| j_{Y}\right\rangle \\
\left\langle F_{Y}|,| J_{Y}\right\rangle\end{array}$ & $\begin{array}{l}\text { Network observables } \\
\text { Cycle observables } \\
\text { Physical observables } \\
\text { Fundamental observables }\end{array}$ \\
\hline
\end{tabular}

of $A^{C}$. Defining the cycle affinities $\left\langle F_{X}\right|=\left\langle f_{X}\right| A^{C}$, we obtain the well-known decomposition of the network EPR,

$$
\sigma_{X}=\left\langle f_{X} \mid j_{X}\right\rangle=\left\langle F_{X} \mid J_{X}\right\rangle
$$

where it is important to notice that the affinity of a cycle $\gamma$ only depends on the rates, $F_{X}(\gamma)=\ln \prod_{e \in \gamma} \frac{w_{e}}{w_{-e}}$. Notice that at both the network and the physical levels we resort to uppercase symbols $J, F$ when we take into account the respective conservation laws (of probability, or of physical quantities). See Table I for a recap on the various pairs of conjugate observables that we will employ though the paper.

The passage from statistical to physical thermodynamics is based on the identification of physical currents as linear combinations of network currents:

$$
\left|j_{Y}\right\rangle=A^{Y}\left|j_{X}\right\rangle
$$

where $A_{y, e}^{Y}=y_{x}-y_{x^{\prime}}$ is the inflow of extensive quantity $y$ as the system performs a transition $e=\left(x x^{\prime}, v\right)$. Steady-state thermodynamic consistency requires that the network EPR $\sigma_{X}$ coincides with the physical one $\sigma_{Y}$, namely there exists a vector of physical thermodynamic affinities $\left\langle f_{Y}\right|$ such that

$$
\sigma_{Y}:=\left\langle f_{Y} \mid j_{Y}\right\rangle=\left\langle f_{X} \mid j_{X}\right\rangle=\left\langle F_{X} \mid J_{X}\right\rangle
$$

Steady-state thermodynamic consistency is granted by the following condition of LDB,

$$
\left\langle f_{X}\right|=\left\langle f_{Y}\right| A^{Y}+\langle\Delta \phi|
$$

on the assumption that $\langle\Delta \phi| A^{C}=0$. This formula then relates the edge affinities to the reservoir entropy changes caused by the transition along that edge, up to an increase in a state function $\phi$ that measures the system entropic changes. We discuss specific examples below.

Introducing the $n_{Y} \times n_{X}$ matrix

$$
M:=A^{Y} A^{C},
$$

we find

$$
\left|j_{Y}\right\rangle=M\left|J_{X}\right\rangle, \quad\left\langle F_{X}\right|=\left\langle f_{Y}\right| M .
$$

This shows that $M$ is the crucial object to understand the mapping between physical and cycle thermodynamics, as it mixes "black box" information and topology. Importantly, the passage from physical to cycle EPR in Eq. (6) comes with a balance of conservation laws and symmetries. On the one hand, letting $\langle w|$ be any of the $\lambda_{Y}$ independent left-null vectors of $M$, then $\left\langle w \mid j_{Y}\right\rangle=0$, which expresses the conservation of physical currents across the system. On the other hand, for each of the $\lambda_{X}$ right-null vectors $|v\rangle$ of $M$ we have $\left\langle F_{X} \mid v\right\rangle=$ 0 , which expresses symmetries of the cycle affinities. Notice that the inverse problem of reconstructing $\left\langle f_{Y}\right|$ from the cycle affinities $\left\langle F_{X}\right|$, for given $\phi$, is not uniquely determined, and, as a consequence, one can further compress the expression for the EPR. Since the rank of $M$ is

$$
\alpha:=n_{Y}-\lambda_{Y}=n_{X}-\lambda_{X}
$$

the EPR can be expressed as

$$
\sigma=\left\langle F_{Y} \mid J_{Y}\right\rangle
$$

in terms of a reduced number $\alpha$ of fundamental physical currents $\left|J_{Y}\right\rangle$ and affinities $\left\langle F_{Y}\right|$. The latter two equations summarize our main findings: The EPR of a system at steady state between different reservoirs with $n_{Y}$ affinities only displays $\alpha=n_{Y}-\lambda_{Y}$ fundamental affinities and currents because the internal structure of the system enforces $\lambda_{Y}$ (i.e., the dimension of the cokernel of $M$ ) conservation laws. We also improve the Schnakenberg construction since the number of symmetries $\lambda_{X}$ (i.e., the dimension of the kernel of $M$ ) determines how many of the $n_{X}$ cycle currents and cycle affinities are redundant. The balance Eq. (10) shows that in a given network, for fixed $n_{X}$ and $n_{Y}$, when varying the reservoir affinities and thus the rates, the eventual appearance of an additional conservation law necessarily comes with the simultaneous appearance of one further symmetry of the affinities in a mechanism that is reminiscent of Noether's theorem in classical mechanics (see Ref. [18] for a different formulation of a Markovian Noether-type theorem for the probability).

Let us emphasize once more an important aspect of the theory. As observed, the right- and left-null spaces of $M$ identify conservation laws and symmetries of the affinities. Matrix $M$ is the product of $A^{Y}$, which follows from the definition of the physical currents, and of $A^{C}$, which expresses a structural constraint on the internal state space of the system. Since every right-null vector of $A^{C}$ is also a null vector of $M$, one might be tempted to speculate that conservation laws and symmetries are only manifestations, respectively, of the physical definition and of the topology of the state space. However, notice that $A^{C}$ is full-rank. Therefore, every symmetry arises as a consequence of the interplay between topology and physics. Similarly, "black box" conservation laws such as those of energy and number of particles, which are the left-null vectors of $A^{Y}$, are only some of the whole set of conservation laws that emerge from the interplay between topology and physics, which are the left-null vectors of $M$.

We now provide a systematic procedure, routine 2 , to produce the fundamental physical affinities and currents. It can be seen as the analog of routine 1 at the physical level: Define $W$ as the matrix of independent left-null vectors of $M$. Notice that $W\left|j_{Y}\right\rangle=0$ implies that $\left|j_{Y}\right\rangle=\tilde{M}\left|J_{Y}\right\rangle$, where $\tilde{M}$ is a matrix of independent right-null vectors of $W$ (e.g., obtained by removing $\lambda_{Y}$ columns from $M$ ). To find the fundamental currents we then just need to invert this relation using the Moore-Penrose pseudoinverse, $\left|J_{Y}\right\rangle=\tilde{M}^{+}\left|j_{Y}\right\rangle=$ $\tilde{M}^{+} M\left|J_{X}\right\rangle$ [19]. Similarly, the fundamental affinities can be found by solving the linear equations $\left\langle F_{Y}\right| \tilde{M}^{+} M=\left\langle F_{X}\right|$ on the subspace $\left\langle F_{X}\right| V=0$, where $V$ is the matrix of right-null vectors of $M$. A vector space analysis shows that this routine has a unique solution (found, for example, by removing $\lambda_{X}$ linear equations corresponding to nonindependent rows of $\left.\tilde{M}^{+} M\right)$. However, like for $A^{C}$, there is freedom in the choice 
of $\tilde{M}$ and the choice of a preferred basis of null vectors must be based on the specifics of the system at hand.

Let us resume our results by the following algorithm, which, for a given model, finds conservation laws and symmetries and provides an expression for the fundamental currents and affinities:

(i) Input master equation with rates satisfying LDB;

(ii) Find the incidence matrix $A^{X}$;

(iii) Find $A^{C}$ using routine 1;

(iv) Calculate cycle affinities $\left\langle F_{X}\right|$ and currents $\left|J_{X}\right\rangle$;

(v) Input $A^{Y}$ compatibly with LDB;

(vi) Compute $M=A^{Y} A^{C}$;

(vii) Find symmetries as right-null vectors of $M$;

(viii) Find conservation laws as left-null vectors of $M$; and

(ix) Use routine 2 to find the fundamental affinities $\left\langle F_{Y}\right|$ and currents $\left|J_{Y}\right\rangle$.

Let us now discuss some examples of LDB, as can be found, e.g., in Refs. [4,20-23]. When the system transitions are caused by exchanges of energy and particles $y=\left(\epsilon_{1}, \ldots, \epsilon_{R}, n_{1}, \ldots, n_{R}\right)$, one at the time, with $R$ grand-canonical reservoirs with physical affinities $\left\langle f_{Y}\right|=$ $\left(\beta^{1}, \ldots, \beta^{R},-\beta^{1} \mu^{1}, \ldots,-\beta^{R} \mu^{R}\right)$, and possibly internal entropy states, e.g., due to coarse graining [4], the LDB condition reads [10]

$$
\ln \frac{w_{x x^{\prime}}^{r}}{w_{x^{\prime} x}^{r}}=\beta^{r}\left(\epsilon_{x^{\prime}}-\epsilon_{x}\right)-\beta^{r} \mu^{r}\left(n_{x^{\prime}}-n_{x}\right)+s_{x^{\prime}}-s_{x} .
$$

Energy and matter currents are given respectively by

$$
\begin{aligned}
& j_{\epsilon_{r}}^{Y}=\sum_{x, x^{\prime}}\left(\epsilon_{x}-\epsilon_{x^{\prime}}\right) j_{x x^{\prime}, r}^{X}, \\
& j_{n_{r}}^{Y}=\sum_{x, x^{\prime}}\left(n_{x}-n_{x^{\prime}}\right) j_{x x^{\prime}, r}^{X} .
\end{aligned}
$$

This setup can be easily shown to satisfy the above framework, with matrix $A^{Y}$ given by

$$
A_{y, e}^{Y}=\left\{\begin{array}{lll}
\epsilon_{x^{\prime}}-\epsilon_{x}, & \text { if } e=x \stackrel{r}{\leftarrow} x^{\prime}, \quad y=\epsilon_{r} \\
n_{x^{\prime}}-n_{x}, & \text { if } e=x \stackrel{r}{\leftarrow} x^{\prime}, \quad y=n_{r} \\
0 & \text { otherwise }
\end{array}\right.
$$

and the potential $\phi_{x}$ accounting for the internal entropy $s_{x}$ and for the self-information $-\log p_{x}$. It follows from the fact that $A^{Y}$ has a block structure (energy or particle) and that it is defined only in terms of energy differences and of particle number differences that the maximum value of $\alpha$ is $2(R-1)$. However, additional symmetries following from the network properties might further reduce this number. They can be deduced neither from the single edge level where the LDB is expressed, nor from a global black-box perspective.

Another example of local detailed balance is the stochastic mass-action kinetics (MAK) in population dynamics. Any occurrence of, say, reaction $2 A \rightarrow B$ decreases the population of $A$ by 2 , independently of the initial population; hence the physical current is $j_{A}=2 \sum_{a} j_{X}(a-2 \leftarrow a)$. Microscopic affinities instead depend on the populations, as, e.g., the rate of an encounter of two molecules is proportional to $a(a-1)$. Still, MAK again satisfies Eq. (12) with potential $\phi$, including the self-information and a factorial term due to particle indistinguishibility, cf. Ref. [20] for details.
We conclude by going back to our model system in light of the full theory. Details are presented in the Appendix. A Schnakenberg analysis reveals $n_{X}=3$ cycle affinities $F_{X}^{1}=\left(\epsilon_{d}+u\right)\left(\beta_{3}-\beta_{2}\right)+\beta_{2} \mu_{2}-\beta_{3} \mu_{3}, F_{X}^{2}=\epsilon_{d}\left(\beta_{2}-\right.$ $\left.\beta_{3}\right)+\beta_{3} \mu_{3}-\beta_{2} \mu_{2}$, and $F_{X}^{3}=\left(\beta_{1}-\beta_{3}\right) u$, corresponding to the three cycles depicted in Fig. 1 . The matrix $M$ reads

$$
M=\left(\begin{array}{ccc}
0 & 0 & u \\
-\epsilon_{d}-u & \epsilon_{d} & 0 \\
\epsilon_{d}+u & -\epsilon_{d} & -u \\
0 & 0 & 0 \\
-1 & 1 & 0 \\
1 & -1 & 0
\end{array}\right)
$$

Since it is full-rank, there is no symmetry of the affinity $\lambda_{X}=0$, which implies that thermodynamic consistency is granted, and that there are $\alpha=3$ fundamental affinities and currents, and $\lambda_{Y}=3$ conservation laws corresponding to left-null vectors of $M$, namely

$$
W=\left(\begin{array}{llllll}
1 & 1 & 1 & 0 & 0 & 0 \\
0 & 0 & 0 & 1 & 0 & 0 \\
0 & 0 & 0 & 0 & 1 & 1
\end{array}\right)
$$

whose rows correspond respectively to total energy conservation, conservation of the number of particles in the upper quantum dot, and conservation of the number of particles in the lower quantum dot. More interesting is the situation when we set all $\beta_{r} \mu_{r}$ identical and thus $n_{Y}=3$. The physical framework then reduces to the fluxes of energy only. Matrix $M$ is given by the upper half-block in Eq. (16). Then, there is one conservation law $W=(1,1,1)$, one symmetry of the affinities $V^{T}=\left(\epsilon_{d}, \epsilon_{d}+u, 0\right)$, and thus $\alpha=2$ fundamental affinities and currents. The fact that $\left\langle F_{X}\right| V=0$ again confirms that LDB grants thermodynamic consistency.

\section{Example: Random grid}

While the above example could be worked out by hand without the aid of the machinery outlined above, first-sight resolutions are impossible when the network becomes large. The potential utility of our approach is illustrated in this section with application to a randomized grid that could in principle be made arbitrarily large and complex.

We consider a network given by a bidimensional and equally spaced grid with $n_{X}$ nodes, in contact with $n_{Y}$ reservoirs, each at a given thermodynamic potential. Transitions are only allowed between nearest neighbors, and each of these generates a physical current.

Each network node has position coordinates $x \equiv\left(x_{1}, x_{2}\right)$ and is connected to each of its nearest neighbors by a reservoir with potential $f_{y}$ through an edge $e_{y}=\left(x x^{\prime}, y\right)_{x<x^{\prime}}$, the ordering being chosen from top to bottom and left to right of the grid. The choice of reservoir involved along a particular edge is random. Each reservoir generates the physical current

$$
j_{y}^{Y}=\sum_{x \sim x^{\prime}} a_{x x^{\prime}} j_{x x^{\prime}}^{y},
$$

the sum being over all pairs of nearest neighbors; $j_{x x^{\prime}}^{y}$ stands for the edge probability current between $x$ and $x^{\prime}$, and $a_{x x^{\prime}}=x_{1}-x_{1}^{\prime}+x_{2}-x_{2}^{\prime}$ is here the amount of physical quantity displaced through the grid by reservoir $y$ when the 


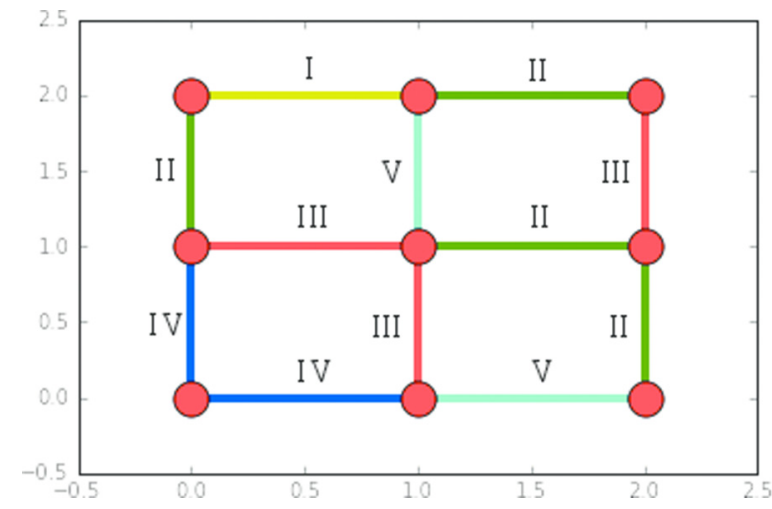

FIG. 2. (a) Schematic representation of our $3 \times 3$ system connected to $n_{Y}=5$ reservoirs. Transitions are allowed between nearest neighbors and the roman numbering of and the colors of edges are associated to the reservoirs involved along the corresponding transitions.

corresponding transition occurs. The matrix $A^{Y}$ transforming the probability current along edges to the physical currents Eq. (18) is thus given by

$$
A_{y, e}^{Y}=\left\{\begin{array}{ll}
a_{x x^{\prime}}, & \text { if } e=x \stackrel{y}{\leftarrow} x^{\prime} \\
0, & \text { otherwise }
\end{array} .\right.
$$

Equation (18) can then be expressed in vector form as $\left|j^{Y}\right\rangle=A^{Y}\left|j^{X}\right\rangle$ in terms of the vectors of physical currents $\left|j^{Y}\right\rangle=\left(j_{1}^{Y}, j_{2}^{Y}, \ldots, j_{n_{Y}}^{Y}\right)$ and probability currents $\left|j^{X}\right\rangle=$ $\left(j_{1}^{X}, j_{2}^{X}, \ldots, j_{n_{e}}^{Y}\right)$, with $n_{e}$ denoting the number of edges on the network. While we do not need to explicitly specify the transition rates of our stochastic process, we assume local detailed balance

$$
\begin{aligned}
\ln \frac{w_{x x^{\prime}}^{y}}{w_{x^{\prime} x}^{y}} & =f_{y}\left(x_{1}^{\prime}-x_{1}+x_{2}^{\prime}-x_{2}\right) \equiv f_{y} a_{x^{\prime} x}, \\
y & =1,2, \ldots, n_{Y} .
\end{aligned}
$$

As discussed in the main text, the balance between the number of symmetries of the thermodynamic affinities and conservation laws is obtained by evaluating the rank of the matrix $M=A^{Y} A^{C}$, where $A^{C}$ denotes the cycle matrix of the network.

A randomly generated $3 \times 3$ grid with five reservoirs is provided in Fig. 2, where each color of the edges is associated to one reservoir. By direct inspection, it is a priori unintuitive how many conservation laws and independent thermodynamic affinities there are. This particular network has $n_{X}=4$ independent cycles, $n_{Y}=5$ physical currents. The rank of the matrix $M$ can be evaluated to yield $\alpha=2$, which is the number fundamental currents and affinities. Thus there are $n_{Y}-\alpha=3$ conservation laws for the physical fluxes $j_{y}^{Y}$ and $n_{X}-\alpha=2$ symmetries of the fundamental affinities under shifts of the forces $f_{y}$.

A posteriori, we can make sense of this result by realizing that, because each transition displaces \pm 1 bit of $x$ through the grid, then the lower-left cycle mediated by reservoirs III-IIIIV-IV has vanishing cycle affinity and that the two cycles on the right both mediated by reservoirs II-III-II-V have the same affinity, which explains the symmetry. However, it is clear that this naked-eye analysis cannot be scaled up with increasing network size.

Therefore, this particular example shows that even for simple topologies, nontrivial conservation laws and symmetries of the fundamental affinities might arise. This property is also quite generic since it emerges in grids whose distribution of currents and forces are randomly chosen. Furthermore, this also illustrates that the fundamental affinities ( 2 in number) are not trivially obtained from the cycle analysis (4 in number). Nontrivial conservation laws, reducing the number of independent thermodynamic forces, emerge even for such simple topology and for a relatively small-sized network. The usefulness of routine 2 is even more apparent for increasing grid size as the identification of the independent thermodynamic forces becomes in any case intractable.

\section{FLUCTUATION THEOREM FOR THE FUNDAMENTAL CURRENTS}

Finally, it is useful to consider the most celebrated result in ST, namely the fluctuation theorem (FT), in light of our theory. Versions of the theorem abound, ranging from the very detailed FT for all of the cycle currents to theorems dedicated to individual physical currents. Here we refer to the formulation found in Refs. [17,24], showing that the FT for the fundamental physical currents hold, provided that the affinities have the due symmetry. Let $\xi_{X}\left(\left\langle Q_{X}\right|\right)$ be the scaled cumulant generating function of the cycle currents, where $\left\langle Q_{X}\right|$ is a vector of counting fields dual to the currents [25]. At the level of the cumulant generating function, the FT takes the form of the Lebowitz-Spohn symmetry

$$
\xi_{X}\left(\left\langle F_{X}\right|-\left\langle Q_{X}\right|\right)=\xi_{X}\left(\left\langle Q_{X}\right|\right) .
$$

The cumulant generating function of the fundamental physical currents $\xi_{Y}$ can be found by the contraction principle according to the theory of large deviations:

$$
\xi_{Y}\left(\left\langle Q_{Y}\right|\right)=\xi_{X}\left(\left\langle Q_{Y}\right| \tilde{M}^{+} M\right) .
$$

Then, the fluctuation relation clearly extends to the physical currents provided that $\left\langle F_{X}\right|=\left\langle F_{Y}\right| \tilde{M}^{+} M$, in which case

$$
\xi_{Y}\left(\left\langle F_{Y}\right|-\left\langle Q_{Y}\right|\right)=\xi_{Y}\left(\left\langle Q_{Y}\right|\right)
$$

\section{CONCLUSIONS}

We provided a systematic procedure to identify the fundamental set of currents and affinities governing the entropy production of a system in contact with multiple reservoirs, thus expanding the toolbox of stochastic thermodynamics with potential applications to arbitrarily large and complex systems. Our theory revealed the fundamental role of the network topology on the thermodynamic description. Our presentation focused on ensemble-averaged EPR, but our results can be directly transferred to fluctuating ST and to fluctuation theorems at the large deviation level $[17,24,26]$.

Finally, as a perspective, let us consider the linear regime. Schnakenberg computed the response matrix $L$ for the cycle observables, showing that it is symmetric and nondegenerate. In our setup, the physical linear response relation reads $\left|f_{Y}\right\rangle=$ $M^{T} L M\left|j_{Y}\right\rangle$, as can be immediately deduced from Eq. (9) and Ref. [11, Eq. (10.18)]. Matrix $M^{T} L M$ is symmetric, hence Onsager symmetry is granted at the macroscopic level in the 
presence of LDB. We notice that the existence of conservation laws is crucial for optimizing the efficiency of machines [27]. Interestingly, the above matrix becomes degenerate in the presence of conservation laws; degeneracy is precisely the condition required to reach the so-called tight-coupling condition that optimizes the efficiency of machines [28]. Therefore our analysis might have interesting consequences in the study of efficiency enhancement.

\section{ACKNOWLEDGMENTS}

This research was supported by the National Research Fund Luxembourg in the frame of Project No. FNR/A11/02 and of PostDoc Grant No. 7982468 and by the European Research Council (Project No. 681456). Discussion with A. Lazarescu and with R. Rao was highly beneficial.

\section{APPENDIX}

We apply the algorithm to our model system of coupled quantum dots, first in the presence of both temperature and chemical potential gradients and then in the presence of only temperature gradients. We do not specify the symmetric part of the rates and hence we do not explicitly calculate the currents.

\section{Energy and particles currents}

(i) We choose rates that satisfy local detailed balance:

$$
\begin{aligned}
& \ln \frac{w_{+1}}{w_{-1}}=\beta_{1}\left(\mu_{1}-\epsilon_{u}\right), \quad \ln \frac{w_{+2}}{w_{-2}}=\beta_{2}\left(\mu_{2}-\epsilon_{d}-u\right), \\
& \ln \frac{w_{+3}}{w_{-3}}=\beta_{3}\left(\mu_{3}-\epsilon_{d}-u\right), \quad \ln \frac{w_{+4}}{w_{-4}}=\beta_{1}\left(\epsilon_{u}+\delta \epsilon-\mu_{1}\right), \\
& \ln \frac{w_{+5}}{w_{-5}}=\beta_{2}\left(\epsilon_{d}-\mu_{2}\right), \quad \ln \frac{w_{+6}}{w_{-6}}=\beta_{3}\left(\epsilon_{d}-\mu_{3}\right) .
\end{aligned}
$$

(ii) The incidence matrix of the graph reads

$$
A^{X}=\left(\begin{array}{rrrrrr}
-1 & 0 & 0 & 0 & 1 & 1 \\
1 & -1 & -1 & 0 & 0 & 0 \\
0 & 0 & 0 & 1 & -1 & -1 \\
0 & 1 & 1 & -1 & 0 & 0
\end{array}\right) \text {. }
$$

(iii) According to Schnakenberg's theory, a convenient cycle matrix of independent null vectors is given by

$$
A^{C}=\left(\begin{array}{rrr}
0 & 0 & 1 \\
1 & 0 & 0 \\
-1 & 0 & 1 \\
0 & 0 & 1 \\
0 & 1 & 0 \\
0 & -1 & 1
\end{array}\right)
$$

(iv) Cycle affinities are given by

$$
\begin{aligned}
& F_{X}^{1}=\left(\epsilon_{d}+u\right)\left(\beta_{3}-\beta_{2}\right)+\beta_{2} \mu_{2}-\beta_{3} \mu_{3} \\
& F_{X}^{2}=\epsilon_{d}\left(\beta_{2}-\beta_{3}\right)+\beta_{3} \mu_{3}-\beta_{2} \mu_{2} \\
& F_{X}^{3}=\left(\beta_{1}-\beta_{3}\right) u .
\end{aligned}
$$

Their conjugate currents are

$$
\begin{aligned}
& J_{X}^{1}=w_{+1} p_{00}^{*}-w_{-1} p_{10}^{*} \\
& J_{X}^{2}=w_{+2} p_{10}^{*}-w_{-2} p_{11}^{*} \\
& J_{X}^{3}=w_{+5} p_{01}^{*}-w_{-5} p_{00}^{*},
\end{aligned}
$$

where $p^{*}$ is the steady state of the master equation.

(v) We consider as physical currents the energy and particle currents

$$
\left|j_{Y}\right\rangle=\left(\begin{array}{c}
-\dot{\epsilon}_{1} \\
-\dot{\epsilon}_{2} \\
-\dot{\epsilon}_{3} \\
-\dot{n}_{1} \\
-\dot{n}_{1} \\
-\dot{n}_{3}
\end{array}\right)
$$

Then matrix $A^{Y}$ is given by

$$
A^{Y}=\left(\begin{array}{cccccc}
-\epsilon_{u} & 0 & 0 & \epsilon_{u}+u & 0 & 0 \\
0 & -\epsilon_{d}-u & 0 & 0 & \epsilon_{d} & 0 \\
0 & 0 & -\epsilon_{d}-u & 0 & 0 & \epsilon_{d} \\
-1 & 0 & 0 & 1 & 0 & 0 \\
0 & -1 & 0 & 0 & 1 & 0 \\
0 & 0 & -1 & 0 & 0 & 1
\end{array}\right),
$$

where the upper half-block is for energy currents to and from the three reservoirs and the lower block is for the matter currents.

(vi) Then matrix $M$ turns out to be

$$
M=\left(\begin{array}{ccc}
0 & 0 & u \\
-\epsilon_{d}-u & +\epsilon_{d} & 0 \\
+\epsilon_{d}+u & -\epsilon_{d} & -u \\
0 & 0 & 0 \\
-1 & 1 & 0 \\
1 & -1 & 0
\end{array}\right)
$$

(vii) Since $M$ has rank 3, there are no symmetries.

(viii) A matrix of conservation laws is

$$
W=\left(\begin{array}{llllll}
1 & 1 & 1 & 0 & 0 & 0 \\
0 & 0 & 0 & 1 & 0 & 0 \\
0 & 0 & 0 & 0 & 1 & 1
\end{array}\right)
$$

(ix) We apply routine 2 . A convenient choice for the matrix $\tilde{M}$ of right-null vectors of $W$ is

$$
\tilde{M}=\left(\begin{array}{rrr}
0 & 0 & 1 \\
1 & 0 & 0 \\
-1 & 0 & -1 \\
0 & 0 & 0 \\
0 & 1 & 0 \\
0 & -1 & 0
\end{array}\right)
$$

Its Moore-Penrose pseuroinverse is

$$
\tilde{M}^{+}=\left(\begin{array}{cccccc}
-1 / 3 & 2 / 3 & -1 / 3 & 0 & 0 & 0 \\
0 & 0 & 0 & 0 & 1 / 2 & -1 / 2 \\
2 / 3 & -1 / 3 & -1 / 3 & 0 & 0 & 0
\end{array}\right) .
$$

Therefore:

$$
\left|J_{Y}\right\rangle=\left(\begin{array}{ccc}
-\epsilon_{d}-u & \epsilon_{d} & 0 \\
-1 & 1 & 0 \\
0 & 0 & u
\end{array}\right)\left|J_{X}\right\rangle
$$


The fundamental forces can be calculated by simply inverting

$$
\left\langle F_{Y}\right|=\left\langle F_{X}\right|\left(\begin{array}{ccc}
-\epsilon_{d}-u & \epsilon_{d} & 0 \\
-1 & 1 & 0 \\
0 & 0 & u
\end{array}\right)^{-1}
$$

\section{Energy currents}

We now consider the more interesting case where the chemical potential differences are set to zero. We thus focus only on energy currents.

(iv') Cycle affinities are given by

$$
\begin{aligned}
& F_{X}^{1}=\left(\epsilon_{d}+u\right)\left(\beta_{3}-\beta_{2}\right) \\
& F_{X}^{2}=\epsilon_{d}\left(\beta_{2}-\beta_{3}\right) \\
& F_{X}^{3}=\left(\beta_{1}-\beta_{3}\right) u .
\end{aligned}
$$

$\left(\mathrm{v}^{\prime}\right)$ As physical currents we consider the energy currents:

$$
\left|j_{Y}\right\rangle=\left(\begin{array}{l}
-\dot{\epsilon}_{1} \\
-\dot{\epsilon}_{2} \\
-\dot{\epsilon}_{3}
\end{array}\right)
$$

Then matrix $A^{Y}$ is

$$
A^{Y}=\left(\begin{array}{cccccc}
-\epsilon_{u} & 0 & 0 & \epsilon_{u}+u & 0 & 0 \\
0 & -\epsilon_{d}-u & 0 & 0 & \epsilon_{d} & 0 \\
0 & 0 & -\epsilon_{d}-u & 0 & 0 & \epsilon_{d}
\end{array}\right) .
$$

(vi') Matrix $M$ is

$$
M=\left(\begin{array}{ccc}
0 & 0 & u \\
-\epsilon_{d}-u & \epsilon_{d} & 0 \\
\epsilon_{d}+u & -\epsilon_{d} & -u
\end{array}\right) .
$$

(vii') There is one symmetry

$$
V=\left(\begin{array}{c}
\epsilon_{d} \\
\epsilon_{d}+u \\
0
\end{array}\right)
$$

We have

$$
\left\langle F_{X}\right| V=0,
$$

therefore the model is thermodynamically consistent.

(viii') There is one conservation law,

$$
W=\left(\begin{array}{lll}
1 & 1 & 1
\end{array}\right),
$$

corresponding to total energy conservation. (ix') We apply routine 2 . A choice of matrix $\tilde{M}$ is

$$
\tilde{M}=\left(\begin{array}{cc}
0 & -1 \\
1 & 0 \\
-1 & 1
\end{array}\right) .
$$

Its Moore-Penrose pseudoinverse is

$$
\tilde{M}^{+}=\left(\begin{array}{ccc}
-1 / 3 & 2 / 3 & -1 / 3 \\
-2 / 3 & 1 / 3 & 1 / 3
\end{array}\right) .
$$

Then:

$$
\left(\begin{array}{c}
J_{Y}^{1} \\
J_{Y}^{2}
\end{array}\right)=\frac{1}{3}\left(\begin{array}{c}
\dot{\epsilon}_{1}+\dot{\epsilon}_{3}-2 \dot{\epsilon}_{2} \\
2 \dot{\epsilon}_{1}-\dot{\epsilon}_{2}-\dot{\epsilon}_{3}
\end{array}\right) .
$$

In terms of the cycle currents:

$$
\left|J_{Y}\right\rangle=\left(\begin{array}{ccc}
-\epsilon_{d}-u & \epsilon_{d} & 0 \\
0 & 0 & -u
\end{array}\right)\left|J_{X}\right\rangle
$$

The fundamental affinities are found by solving the system of linear equations

$$
\left\langle F_{Y}\right|\left(\begin{array}{ccc}
-\epsilon_{d}-u & \epsilon_{d} & 0 \\
0 & 0 & -u
\end{array}\right)=\left\langle F_{X}\right| .
$$

We can simply remove the second column of the matrix to obtain the invertible system

$$
\left\langle F_{Y}\right|\left(\begin{array}{cc}
-\epsilon_{d}-u & 0 \\
0 & -u
\end{array}\right)=\left[\begin{array}{c}
\left(\epsilon_{d}+u\right)\left(\beta_{3}-\beta_{2}\right) \\
\left(\beta_{1}-\beta_{3}\right) u
\end{array}\right] .
$$

which gives

$$
\begin{aligned}
& F_{Y}^{1}=\beta_{2}-\beta_{3} \\
& F_{Y}^{2}=\beta_{3}-\beta_{1} .
\end{aligned}
$$

Finally, we obtain for the entropy production rate:

$$
\begin{aligned}
\sigma_{Y}= & \frac{1}{3}\left[\left(\beta_{2}-\beta_{3}\right)\left(\dot{\epsilon}_{1}+\dot{\epsilon}_{3}-2 \dot{\epsilon}_{2}\right)\right. \\
& \left.+\left(\beta_{3}-\beta_{1}\right)\left(2 \dot{\epsilon}_{1}-\dot{\epsilon}_{2}-\dot{\epsilon}_{3}-\right)\right] .
\end{aligned}
$$

Notice that this expression is equivalent to Eq. (2) once the conservation law $\dot{\epsilon}_{1}+\dot{\epsilon}_{2}+\dot{\epsilon}_{3}=0$. This also shows that the procedure we outlined finds one possible choice of conjugate fundamental observables, which might not necessarily be the preferred or more elegant choice for the system of interest. The problem of finding "natural" basis for vector spaces of the currents is a widespread problem, e.g., in the biochemical modeling of metabolic networks.
[1] U. Seifert, Rep. Prog. Phys. 75, 126001 (2012).

[2] C. Van den Broeck and M. Esposito, Physica A 418, 6 (2015).

[3] C. Jarzynski, Annu. Rev. Condens. Matter Phys. 2, 329 (2011).

[4] M. Esposito, Phys. Rev. E 85, 041125 (2012).

[5] B. Altaner and J. Vollmer, Phys. Rev. Lett. 108, 228101 (2012).

[6] M. Einax and A. Nitzan, J. Phys. Chem. C 118, 27226 (2014).

[7] S. R. De Groot and P. Mazur, Non-Equilibrium Thermodynamics (Wiley, New York, 2013).

[8] I. Prigogine, Introduction to Thermodynamics of Irreversible Processes (Interscience, New York, 1967).
[9] C. Maes, K. Netočný, and B. Wynants, Markov Proc. Rel. Fields. 14, 445 (2008).

[10] M. Esposito and C. Van den Broeck, Phys. Rev. E 82, 011143 (2010).

[11] J. Schnakenberg, Rev. Mod. Phys. 48, 571 (1976).

[12] M. Polettini and M. Esposito, J. Chem. Phys. 141, 024117 (2014).

[13] P. Strasberg, G. Schaller, T. Brandes, and M. Esposito, Phys. Rev. Lett. 110, 040601 (2013).

[14] G. Bulnes Cuetara, M. Esposito, and P. Gaspard, Phys. Rev. B 84, 165114 (2011). 
[15] R. Sánchez and M. Büttiker, Phys. Rev. B 83, 085428 (2011).

[16] M. Polettini, Lett. Math. Phys. 105, 89 (2015).

[17] D. Andrieux and P. Gaspard, J. Stat. Phys. 127, 107 (2007).

[18] J. C. Baez and B. Fong, J. Math. Phys. 54, 013301 (2013).

[19] A. Ben-Israel and T. N. E. Greville, Generalized Inverses (Springer-Verlag, Berlin, 2003).

[20] M. Polettini, A. Wachtel, and M. Esposito, J. Chem. Phys. 143, 184103 (2015).

[21] G. Schaller, T. Krause, T. Brandes, and M. Esposito, New J. Phys. 15, 033032 (2013).
[22] G. Bulnes Cuetara, A. Engel, and M. Esposito, New J. Phys. 17, 055002 (2015).

[23] G. Bulnes Cuetara and M. Esposito, New J. Phys. 17, 095005 (2015).

[24] M. Polettini and M. Esposito, J. Stat. Mech. (2014) P10033.

[25] A. Wachtel, J. Vollmer, and B. Altaner, Phys. Rev. E 92, 042132 (2015).

[26] J. L. Lebowitz and H. Spohn, J. Stat. Phys. 95, 333 (1999).

[27] G. Benenti, G. Casati, and J. Wang, Phys. Rev. Lett. 110, 070604 (2013).

[28] F. Jülicher, A. Ajdar, and J. Prost, Rev. Mod. Phys. 69, 1269 (1997). 